\title{
ANÁLISIS NEO-BIBLIOMÉTRICO DE LAS INVESTIGACIONES DE TESIS EN LA ESCUELA ACADÉMICO-PROFESIONAL DE PSICOLOGÍA UNMSM
}

\author{
Alberto Quintana Peña*
}

\begin{abstract}
RESUMEN
Se realiza un estudio neo-bibliométrico de las tesis de pre-grado sustentadas en la EAP de Psicología de la Universidad Nacional Mayor de San Marcos del periodo 2000 al 2005, realizando un análisis pormenorizado del corpus de datos con el Formato de Evaluación de los Reportes de Investigación $\mathrm{FORI}^{1}$, para responder a la interrogante $¿ D e$ qué forma se efectúan las investigaciones para optar el título profesional en la EAP de Psicología de la UNMSM? La base de datos así obtenida fue procesada con el estadístico $\times^{2}$ del programa SPSS 0.12, llegándose, entre otras, a las conclusiones siguientes: en la muestra predominan las tesis de «investigación básica fáctica», en oposición a los «estudios de caso» y a las «monografías». Los temas específicos mayormente tratados son los referidos a «los procesos psicológicos básicos del área cognitiva, el rendimiento académico y el comportamiento sexual». Es de resaltar que a diferencia de los otros enfoques psicológicos, quienes investigaron procesos psicológicos básicos con un enfoque cognitivo-conductual han desarrollado estudios «tecnológico-instrumentales y tecnológico-procedimentales», lo cual permite distinguir a los seguidores de este enfoque por su especial interés en la investigación aplicada. Las fuentes de información mayormente usadas como referencias son «secundarias» y están escritas en español. Respecto a la población más frecuentemente estudiada está constituida por adolescentes (estudiantes de secundaria y universitarios), limeños y de clase media.
\end{abstract}

Palabras clave: Estudio neobibliométrico, tesis de pregrado, ciencia, investigación.

\begin{abstract}
It is carried out a study neo-bibliometrico of the pre-grade theses sustained in the EAP of Psychology of the Unversidad Nacional Mayor de San Marcos during the period 20002005, carrying out an itemized analysis of the corpus of data with the Format of Evaluation of the Reports of Investigation FORI, to respond to the query How the investigations are made to opt the I title professional in the EAP of Psychology of the UNMSM? The database this way obtained it was processed with the $\times^{2}$ statistical program SPSS 0.12 , arriving to the following summations: In the sample predominate investigate thesis basicfeasible, opposite to «case's study» and «essay». The specific topics usually are «psychological
\end{abstract}

* Docente Principal de la Facultad de Psicología de la UNMSM.

E-mail: aquintanap@unmsm.edu.pe.

1 El autor agradece la colaboración en la elaboración de este artículo de la Psicóloga Lizeth Rubio. 
processes, about the cognition area, academic achievement and sexual behavior». The egresados that you/they investigated the topic of the basic psychological processes with a cognitive-behavioral focus has developed studies Frequently they were used like references sources of information The frequently studied population the adolescents conform (students of secondary and university students), Limean and of middle class.

Keywords: Study neo-bibliomètrico, Pre-grade thesis, Science, Investigation.

\section{INTRODUCCIÓN}

La investigación resulta ser un componente muy importante en el desarrollo de los programas de formación académico-profesional brindados en la universidad. Se establece como un factor necesario que debe desarrollarse mediante dos vertientes principales: el quehacer de la facultad y el de los estudiantes, (Maura, M., Mercado, J. y Aristeguieta, S., 2004). Desde la perspectiva de la facultad se manifiesta en la realización de iniciativas que respondan a la vez a los objetivos del programa de formación académico-profesional y a los intereses de los facultativos. Desde el ángulo estudiantil se canaliza mayormente a través de las tesis de grado y de Título.

En la Universidad Nacional Mayor de San Marcos (UNMSM), la tesis es una de las modalidades para optar el Título Profesional de Psicólogo. En este contexto, el estudio presentado como tesis para la obtención del título Profesional debe ser para el (la) egresado(a) un ejercicio por el cual éste demuestra el dominio de destrezas de investigación y la capacidad para el trabajo heurístico en un determinado campo de estudio. En la sustentación, el (la) estudiante deberá demostrar la pertinencia científica y profesional del tema a estudiarse, el conocimiento y disponibilidad de fuentes de información (referencias bibliográficas) significativas, el manejo eficaz de estrategias de investigación propias de la disciplina o área de estudio, así como de instrumentos y técnicas de recolección de información validos y confiables. La normatividad institucional prevé que con la aprobación del proyecto de tesis se garantice que el (la) estudiante se embarque en la investigación con un plan concreto de trabajo y que el programa de formación académico-profesional, el Jurado de tesis, y su asesor de tesis así como la comunidad universitaria tengan constancia de ello (Reglamento de Grados y Titulos, 1993)

Durante el ejercicio de nuestra actual gestión en la dirección de la Escuela Académicoprofesional (EAP) de Psicología de la UNMSM, transcurrida en tiempos en los que en nuestra casa de estudios y la comunidad universitaria nacional se hacen cada vez más consensuadas corrientes de opinión favorables a la autoevaluación con fines de mejora de las instituciones académicas y los programas de formación académico-profesional, consideramos imperativo estimar algunos indicadores de logro del currículo vigente, por ello, entre otras acciones, nos propusimos hacer un análisis pormenorizado y objetivo de las tesis aprobadas en la EAP de Psicología desde el año 2000 hasta el 2005. En el desempeño de este compromiso utilizamos como metodología de trabajo el Análisis Neobibliométrico del corpus de datos en referencia, metodología que nos permite analizar empíricamente la actividad científica generada por los postulantes al título (Quintana y Ojeda, 1996; Pulgarín, Carapeto, y Cobos, 2004). Con tal propósito utilizamos como instrumento para la recolección estructurada de la información el Formato de Evaluación de los Reportes de Investigación FORI (Quintana, 1995). 
En este punto resulta conveniente explicitar que el enfoque neobibliométrico conlleva el uso y la aplicación de métodos cuantitativos (indicadores y modelos matemáticos) a la literatura científica, con el objeto de obtener datos que puedan aportar conocimiento acerca de la evolución de una determinada producción científica, conocer su calidad y obtener elementos de juicio para posibles intervenciones o interpretaciones de hechos o fenómenos sociales (Pulgarín, Carapeto, y Cobos, 2004). Así, la Cienciometría o Cientimetría (Gardfield, 1995) también denominada por otros Metodología Bibliométrica (Carpintero y Peiro, 1981), es ante todo una estrategia metodológica inicialmente centrada en el análisis estructural y prognosis de la información contenida, principalmente, en publicaciones científicas de investigación.

Resulta claro para quienes están familiarizados con la bibliometría que, como ya ha señalado Carpintero (1981), la mejor forma de comprender la urdimbre y multiplicidad de la ciencia consiste en abordarla como una organización. En tanto la diversidad de la ciencia no impide visualizar su «unidad teleológica», pues a pesar de ella, por el entramado que articula el sistema de finalidades de la ciencia, un fin permanente de ésta es su objetivo de descubrir leyes y regularidades en los fenómenos y procesos. Considerando los propulsores de esta metodología que es a través de esta dinámica estructurada y a la vez estructurante de la ciencia, que pueden recorrerse sus dimensiones organizacionales.

Al respecto Carpintero (1981, pp. 32), nos refiere «El propósito, al postular una consideración de la ciencia como organización es lograr sentar las bases de una consideración coherente entre dimensiones cualitativas, teóricas, conceptuales, y otras cuantitativas, materiales y sociales, que aparecen siempre que se considera la compleja realidad de la ciencia moderna».

La comunicación, en efecto, es el medio que permite el proceso correctivo, la discusión y, eventualmente, la inclusión de los resultados obtenidos en el patrimonio intelectual de una comunidad científica relevante (Price, 1978). Precisamente por ello hay autores como Carpintero (1981, pp 34) que señalan:

«...el estudio de la comunicación, esencial para descubrir la estructura organizacional, nos parece un camino particularmente válido en el caso de la ciencia (...) el análisis detallado del cuerpo de comunicaciones, y a su través, de los aspectos organizacionales relevantes, es una vía necesaria en la aproximación a una imagen de ciencia. Es la razón por la que hemos venido aplicando una investigación con metodología bibliométrica en la ciencia, y más en concreto, a la psicología».

Dicha metodología bibliométrica ha sido definida como la cuantificación de la información bibliográfica que resulta susceptible de ser analizada (Garfield et al., 1978) y como el estudio cuantitativo y el análisis de aquellas dimensiones de los materiales bibliográficos que permiten su medición. (Carpintero y Tortosa, 1990).

En este contexto, Carpintero (1981) nos va a decir que la bibliometría estudia la naturaleza y el curso de una disciplina (en tanto que dé lugar a publicaciones) por medio del cómputo y análisis de las varias facetas de la comunicación escrita. Siendo sus objetivos fundamentales, por una parte, el estudio del tamaño, crecimiento y distribución de los documentos científicos y, por otra, la indagación de la estructura y dinámica de los grupos que producen y consumen dichos documentos y la información que contienen.

En 1996, al referirse al método neobibliométrico desde una óptica de gestión del conocimiento, Quintana y Ojeda consideran que sus resultados podrán permitir «... a los nuevos investigadores 
insertar su actividad en un contexto sobre el cual se encuentren informados; así como posibilitar a los administradores respectivos la planificación de políticas de investigación que conllevan a un mejoramiento de la calidad, excelencia y utilidad de la actividad científica» (p. 35).

En 1999, Alcaide Gonzales refiere: en los ensayos bibliométricos se deben integrar «los estudios estadísticos» a «los de carácter social», dotando, de esta manera «los diversos estudios de una característica interdisciplinar indispensable para el desarrollo de los diferentes análisis a los que se someten las distintas fuentes».

Y en el 2000 Antonio Algaba va a realizar un análisis del desarrollo de las revistas científicas españolas, que le permitió conocer la información de que disponían los científicos, técnicos e intelectuales en cada periodo histórico y territorio; así como conocer cuando se inician los distintos debates científicos que han sido determinantes en la evolución de las diferentes especialidades científicas en este país. De la misma manera Algaba va a afirmar que el análisis de la producción de revistas científicas también permite observar el proceso local de especialización del conocimiento, pues la publicación de una revista especializada sería un claro símbolo de la madurez alcanzada por una comunidad-disciplina científica en un país concreto.

\section{MÉTODOS}

Como en lo sucesivo consideramos a las reportes de investigación como unidades de análisis, a fin de no sólo realizar el análisis del tamaño, crecimiento y distribución de la bibliografía científica y la estructura social de los grupos que la producen como lo hace el método bibliométrico (Price, 1973), sino, de enriquecer el mismo analizando también los enfoques, métodos y poblaciones muestrales que los científicos utilizan, bien podríamos convenir en denominar a esta variedad del Método reseñado como método Neo Bibliométrico (Quintana, 1996).

En este estudio se aborda a las tesis de investigación en su condición de reportes de investigación sustentados por los egresados de una carrera académico-profesional a fin de optar el título profesional, es decir, de reportes de investigación sustentados por los egresados con el objetivo de demostrar que cuentan con las competencias conceptuales, procedimentales y actitudinales necesarias y suficientes para merecer que la universidad los acredite como profesionales.

Consideramos que este estudio constituye un importante aporte para dar a conocer las diferentes áreas de trabajo, escuelas teóricas, metodologías, variables, poblaciones, etc, de interés para nuestros aún pocos egresados que optaron el título profesional por tesis de investigación en los últimos años y, sobre todo, para levantar un perfil del académicoinvestigador que viene siendo formado por el currículo vigente de la Carrera académicoprofesional de Psicología en la UNMSM.

Con este objetivo nos proponemos responder a las siguientes interrogantes:

1. ¿De qué forma se efectúan las investigaciones para optar el Título Profesional por Tesis en la UNMSM?

2. ¿Qué temas específicos se estudian habitualmente? 
3. ¿Qué metodologías se usan habitualmente en las investigaciones?

4. ¿De qué tipo es la información que les sirve de fundamento a las tesis de investigación?

5. ¿Qué grupos poblacionales son los habitualmente estudiados?

\section{Muestra}

Creemos que los antecedentes citados en la introducción muestran la pertinencia de esta metodología para hacer un análisis empírico de la actividad científica, si bien en este trabajo nuestras pretensiones son mucho más modestas que la de los autores citados, pues sólo pretendemos un acercamiento empírico objetivo a la actividad científica desrrollada por los postulantes al título en la EAP de Psicología del 2000 al 2005, por lo cual nuestro corpus de datos los constituyen las tesis aprobadas en la EAP de Psicología en el citado periodo. Recordemos que la EAP de Psicología hace explícito el objetivo de la formación académico-profesional que imparte cuando al referirse al PERFIL PROFESIONAL DEL PSICÓLOGO en su currículo vigente afirma: «...el psicólogo el científico del comportamiento humano, cuyo objetivo dentro de la sociedad es contribuir al desarrollo del potencial psíquico del individuo para que este logre mejores condiciones de vida mediante relaciones interpersonales armoniosas, ...» (EAP de Psicología, 1996).

Así mismo, al referirse a las normas para obtener el Título Profesional prescribe:

«Una vez declarado Expedito al Título el postulante, deberá solicitar alguna de las siguientes modalidades:

a) Por tesis.

b) Por Caso Psicológico.

c) Por Acreditación de tres años de prestación de servicios en labores propias de la profesión del Psicólogo.

d) Por examen de titulación al que podrán acceder en forma directa los bachilleres...» (EAP de Psicología, 1993 )

En tanto la modalidad de acreditación requiere la elaboración y sustentación de un estudio monográfico en relación a la práctica para-profesional acreditada, resulta que las tres primeras modalidades ameritan la elaboración de un estudio susceptible de ser sustentado. Estudios cuyos reportes, tesis, se encuentran en la biblioteca de la Facultad de psicología y en la biblioteca central de la UNMSM y que se constituyeron en nuestra muestra de investigación.

Esta muestra de 32 tesis obtenida en forma no-probabilística e intencional fue estudiada mediante un diseño de investigación descriptivo simple (Castro, 1980; Kerlinger, y Howar, 2001; Arnau, 1986; Hernández, Fernández, y Baptista, 1996)

Como instrumento para la recolección de la información se utilizó el Formato de Evaluación de los Reportes de Investigación FORI (Quintana, 1995).

El FORI cubre la mayor parte de aspectos neobibliométricos como las metodologías, temáticas, poblaciones investigadas, fuentes documentales consultadas, así como las formas de asociación de los investigadores, de manera que posibilita tener una concepción analítica 
de las condiciones en las cuales se han realizado la investigaciones que se reportan. El registro de las características de los estudios analizados se realiza a través de códigos o claves, lo cual facilita el manejo de la información, en tanto incrementa la cantidad de datos que se pueden analizar y, a la vez, permite una mayor objetividad sustentada en una estructuración rigurosa de los mismos.

\section{Procedimiento}

El procedimiento comprendió las siguientes etapas: Adaptación y actualización del formato de observación de reportes de investigación, observación y registro en el FORI adaptado de cada una de las 32 tesis, elaboración de la base de datos correspondiente, tratamiento estadístico de los datos con frecuencias, porcentajes y estimación de su significación con $\times^{2}$ e interpretación y discusión de los resultados.

A fin de compartir con el lector los supuestos conceptuales considerados en el instrumento utilizado, FORI, y facilitarle el acceso al significado de los resultados cuantitativos que exponemos a continuación, consideramos ilustrativo presentar, a partir de este punto, los mapas conceptuales que subyacen a la forma en que el instrumento en referencia organiza el registro de información de cada unidad de análisis. Comenzaremos con el mapa conceptual de la Figura 1 que ilustra las relaciones conceptuales que el instrumento establece entre los Tipos de Tesis, los Tipos de Investigación Científica, y el Área Temática y Enfoque de la Psicología en los cuales el egresado enmarca su estudio.

Figura 1. Modalidad de tesis, tipo de investigación, enfoque psicológico de referencia y área temática estudiada.

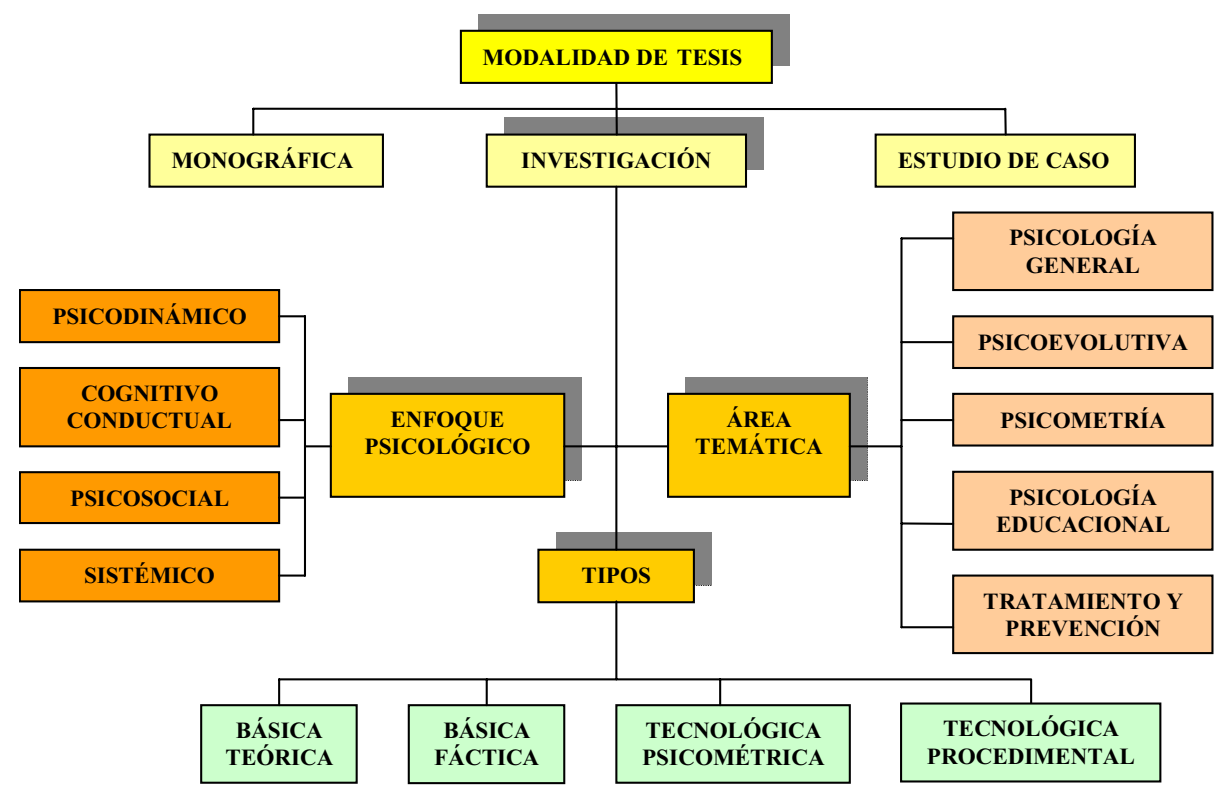




\section{RESULTADOS}

Luego de analizar estadísticamente todos los indicadores que permite registrar el FORI, se estiman resultados estadísticamente significativos solamente en los indicadores siguientes:

\section{Tipo de tesis}

Se observa que en la muestra predominan las 19 tesis de investigación, en oposición a las 9 referidas a monografías y a las tan sólo cuatro de estudios de caso. Razón por la cual en los resultados siguientes nos referiremos sobre todo a las tesis de investigación que constituyeron el $59.38 \%$ de la muestra estudiada con una significación estadística de $\times^{2}=0.004$ (Gráfica 2).

De otro lado se observa en el 2001 y años siguientes un predominio de las tesis de investigación sobre las otras modalidades de acceso al Título profesional, datos estimados con una significación estadística de $\times^{2}=0.210$ (Gráfica 1).

Gráfico 1. Tipo de tesis por año de sustentación.

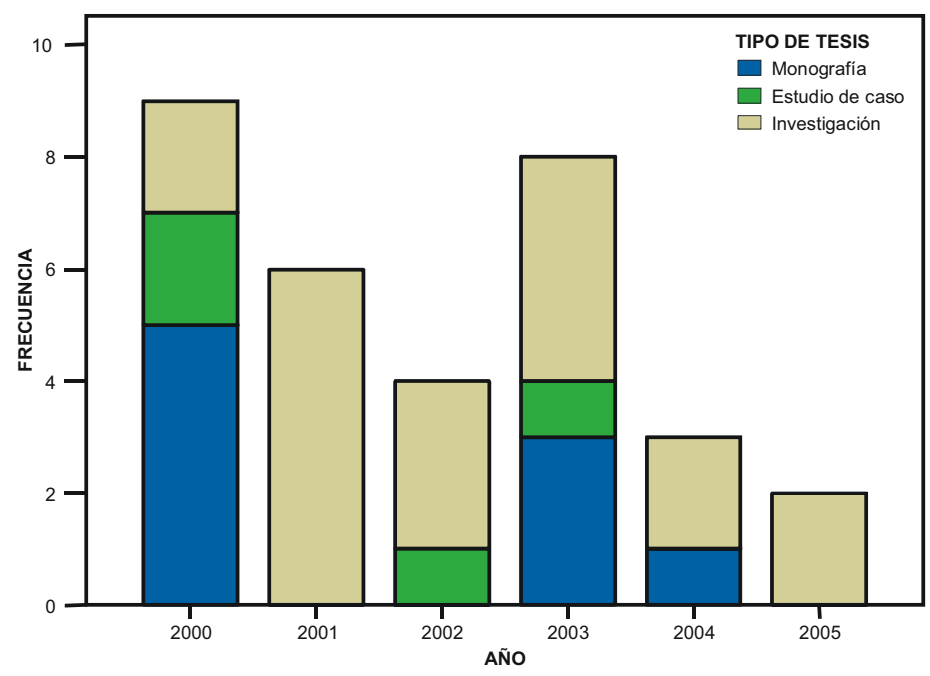

Gráfico 2. Tipo de tesis.

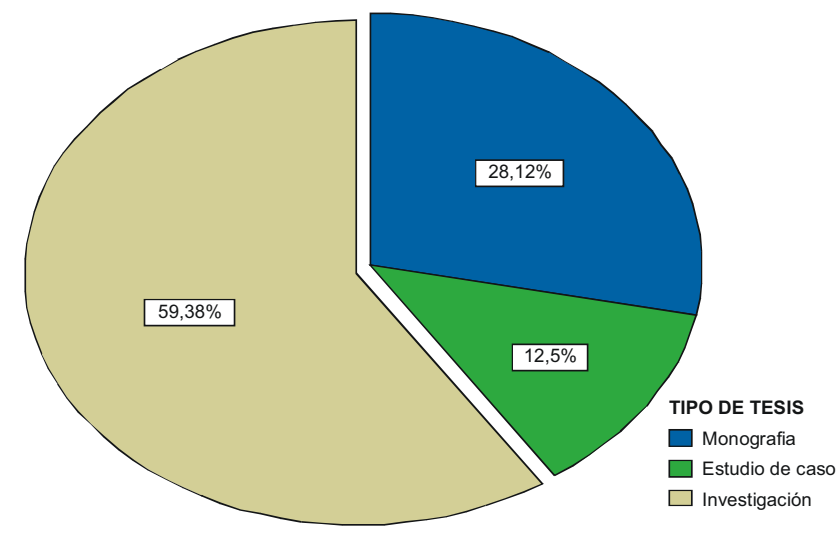




\section{Tipo de investigación}

Como se puede observar en el mapa conceptual precedente se distinguen cuatro tipos de investigación resultantes de considerar si la información es Básica, es decir, busca describir y explicar los hechos y fenómenos del campo de estudio psicológico, en cuyo cometido se aboca a contrastar hipótesis con la realidad a fin de probar su veracidad, en cuyo caso asume el adjetivo de Fáctica, o bien sólo se enfrasca en discusiones conceptuales a partir de supuestos teóricos o resultados de investigaciones antecedentes a fin de demostrar la valides de sus conjeturas, en cuyo caso asume el adjetivo de Teórica. De otro lado se encuentran las investigaciones de tipo Tecnológico, es decir, aquellas que buscan estimar la eficacia y eficiencia de los procedimientos, técnicas e instrumentos utilizados en el ejercicio profesional de la disciplina, distinguiendo aquellas centradas en probar que ciertos procedimientos de intervención producen la modificación prevista de determinados procesos psicológicos o conductas en cuyo caso asume el adjetivo de Procedimental, o bien estimar las «propiedades psicométricas» (Kerlinger, 2001, p. 581) de ciertas técnicas o instrumentos de recolección de información, en cuyo caso asume el adjetivo de Psicométrica.

En el gráfico 3 se puede observar una evidente predominancia de las investigaciones básicas fácticas $68.42 \%$, seguidas de las investigaciones tecnológicas psicométricas en un $21.05 \%$, estando poco representadas, con un $10.53 \%$, las investigaciones de tipo tecnológico procedimental, a desmedro de su importancia para asegurar que el servicio profesional brindado por los profesionales de la psicología es de calidad. Estos datos fueron estimados con una significación estadística de $\times^{2}=0.004$.

Gráfico 3. Tipo de investigación.

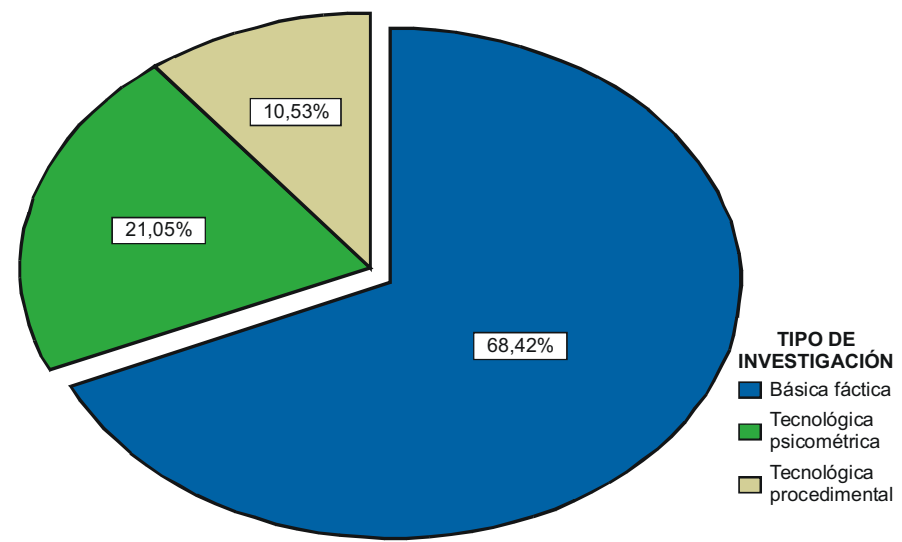

\section{Áreas temáticas investigadas}

El repertorio de áreas temáticas consideradas en el estudio corresponden al presentado en la propuesta original del FORI (Quintana, 1995), por lo cual sólo nos resta reseñar que como se observa en el gráfico 4 , datos estimados con una significación estadística de $\times^{2}=$ 0.165 , es evidente el predominio de investigaciones inscritas en la temática de Psicología Educacional 36.84\%, seguida algo lejos por las referidas a la Psicología Social 21.05, Tratamiento y Prevención $15.79 \%$, y a la Psicología General $15.79 \%$, con lo que se cubre más del $90.00 \%$ de la muestra estudiada. 
Gráfico 4. Áreas temáticas estudiadas.

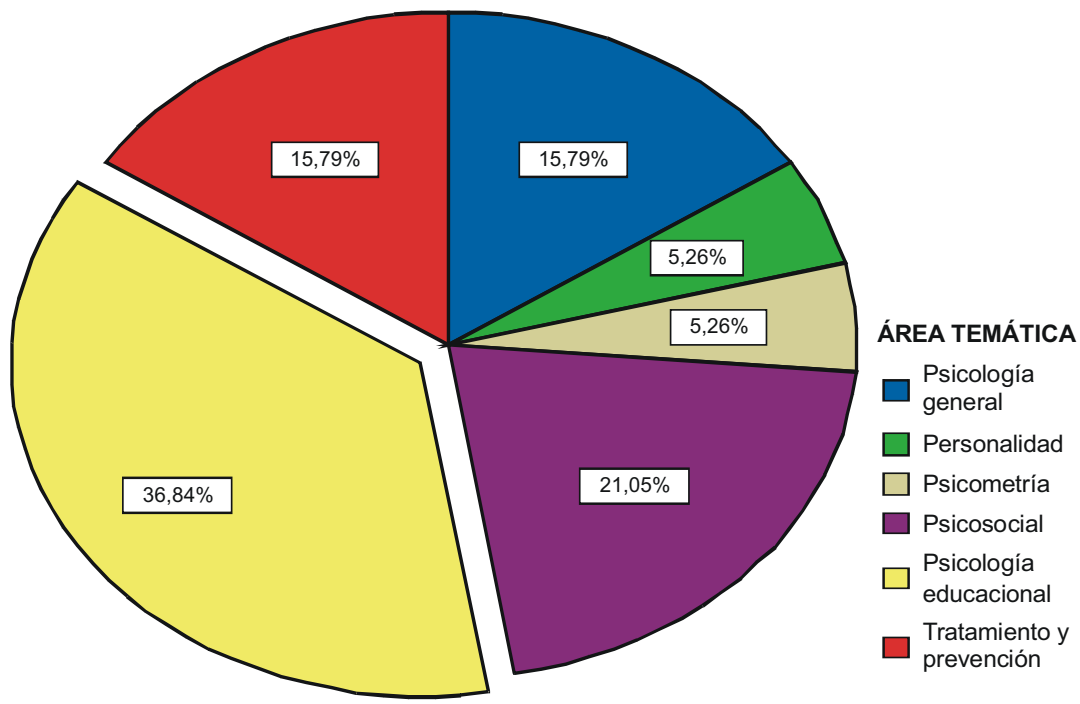

Gráfico 5. Temas específicos estudiados.

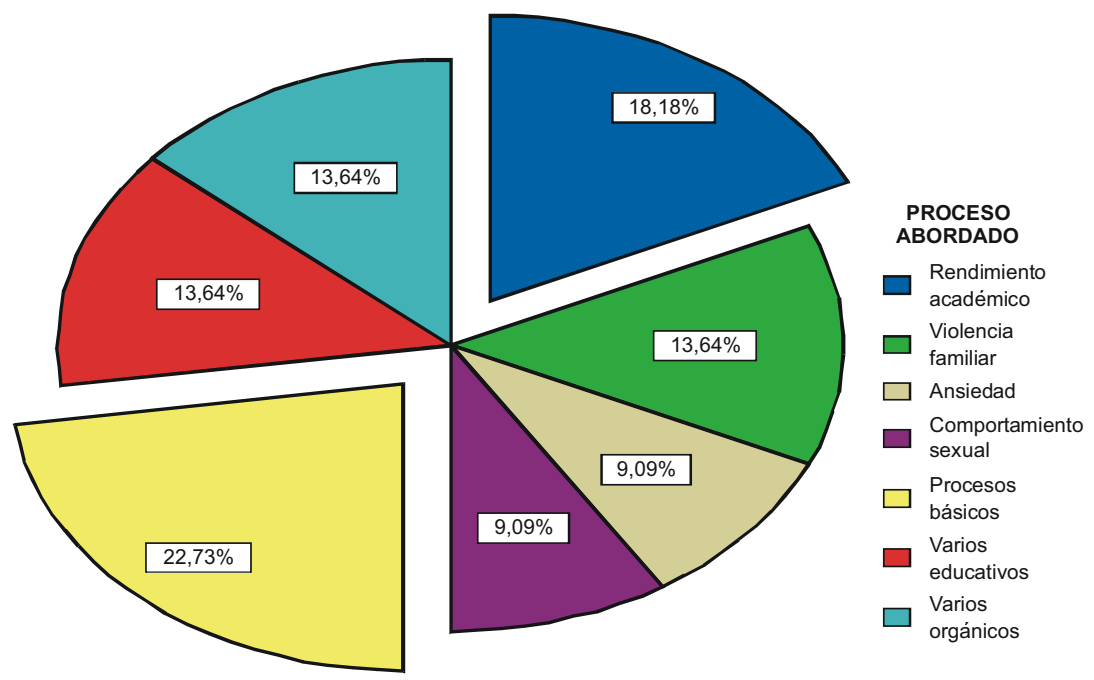


Gráfico 6. Tema específico estudiado por enfoque psicológico.

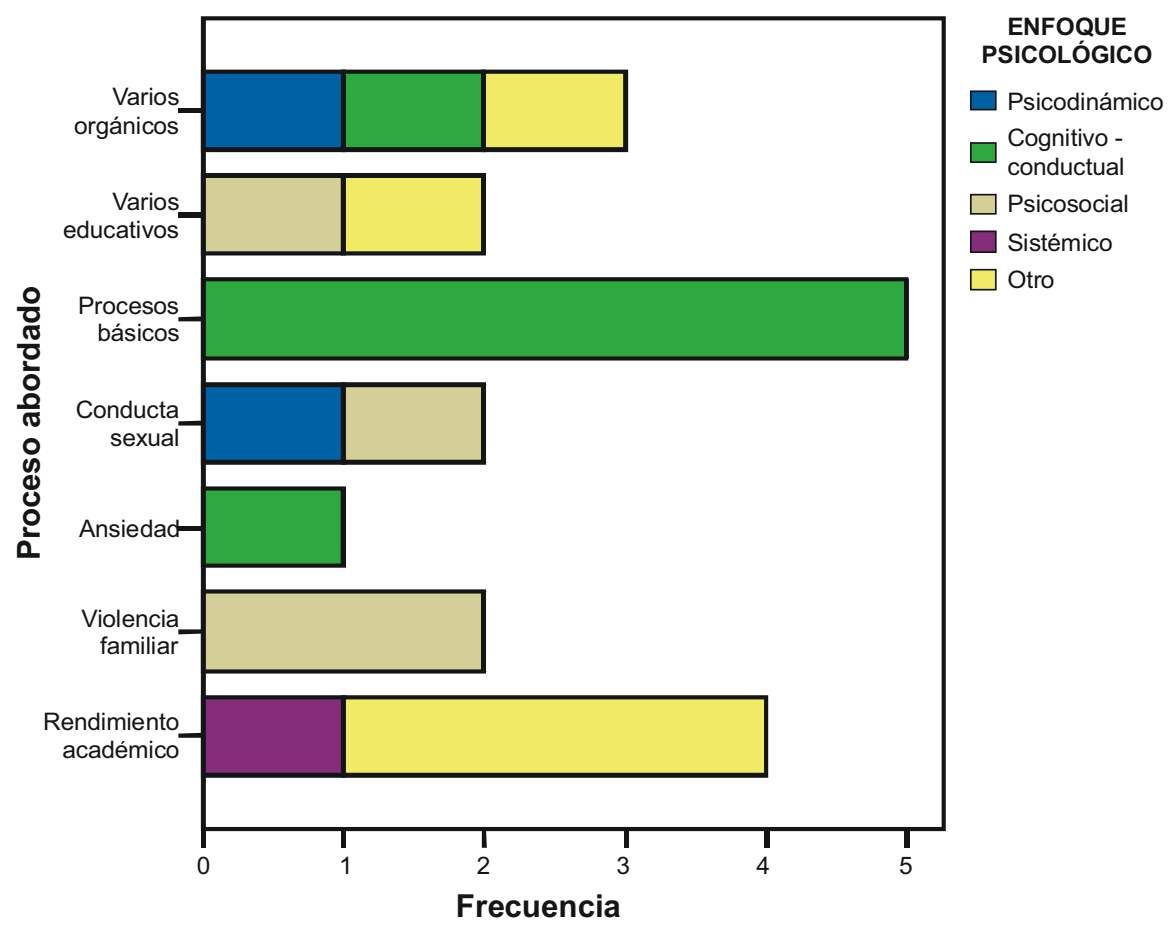

\section{Temas específicos estudiados}

En cuanto a los temas específicos estudiados, datos estimados con una significación estadística de $\times^{2}=0.648$, podemos observar (Grafica 5) una predominancia relativa tesis referidas a los Procesos Básicos $22.73 \%$ y al Rendimiento Académico $18.18 \%$, seguida de las que abordan los varios educativos $13.64 \%$, violencia familiar $13.64 \%$, y varios biomédicos 13.64\%, estando al final las investigaciones que estudian la Ansiedad $9.09 \%$ y el Comportamiento Sexual $9.09 \%$.

Abundando en el análisis observamos en el gráfico 6, acerca del enfoque psicológico en relación al tema especifico estudiado, datos estimados con una significación estadística de $\times^{2}=0.046$, una indiscutible predominancia del Enfoque Cognitivo-Conductual para abordar el estudio de temas acerca de los Procesos Básicos 100\% y la Ansiedad 100\% y del enfoque Psicosocial 100\% para abordar el estudio del tema de Violencia Familiar 100\% 
Figura 2. Nivel y método de la investigación, técnica de muestreo y análisis estadístico utilizado.

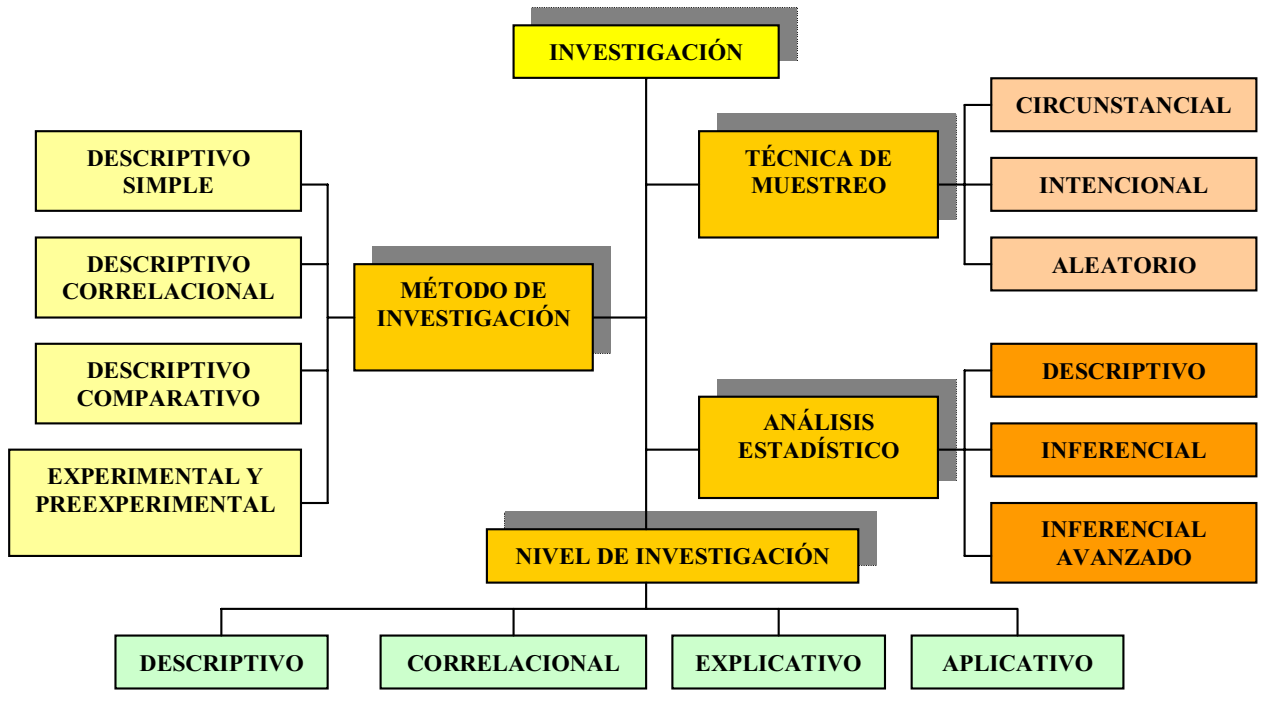

\section{Metodología del estudio}

Comenzaremos este apartado con el mapa conceptual de la Figura 2 que ilustra las relaciones conceptuales que el instrumento establece entre los Niveles de Investigación, Método de Investigación, Técnica de Muestreo y Análisis Estadístico.

En cuanto a los Niveles de Investigación estudiados, datos estimados con una significación estadística de $\times^{2}=0.901$, podemos observar (Gráfico 7) una predominancia del nivel Correlacional $31.58 \%$ seguido por el nivel Descriptivo $26.32 \%$ y por los otros dos niveles con $21.05 \%$ cada uno. De otro lado en el gráfico 8 , acerca del enfoque psicológico en relación al Nivel de Investigación en el que se realiza el estudio, datos estimados con una significación estadística de $\times^{2}=0.005$, una indiscutible predominancia del Enfoque Cognitivo-Conductual en las Investigaciones Aplicadas $100 \%$ y del enfoque Psicosocial $80 \%$ en las Investigaciones de nivel descriptivo.

Gráfico 7. Figura de investigación.

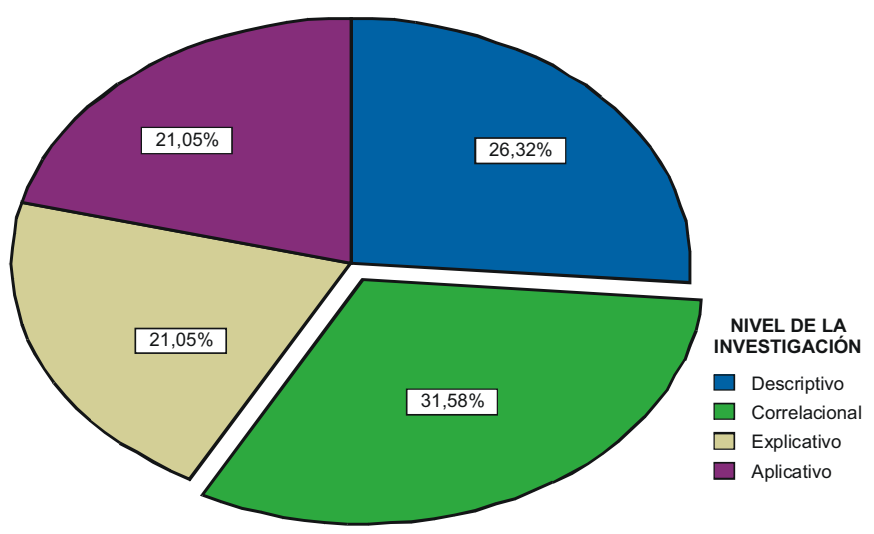


Gráfico 8. Nivel de investigación por enfoque psicológico.

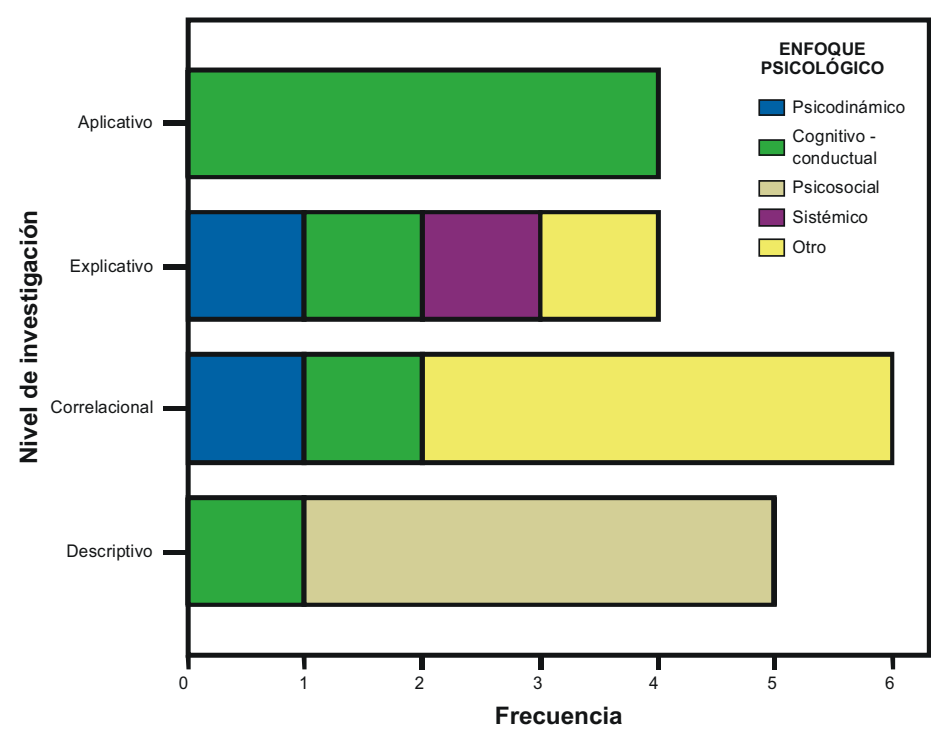

\section{Técnicas de muestreo utilizadas}

En cuanto a las Técnicas de muestreo utilizadas, datos estimados con una significación estadística de $\times^{2}=0.002$, podemos observar (Gráfico 9) una evidente predominancia de las técnicas de muestreo no probabilísticas 78.95\% (Muestreo circunstancial e intencional) sobre las no probabilísticas $21.05 \%$ (Muestreo Aleatorio simple y estratificado).

Gráfico 9. Técnica de muestreo en la investigación.

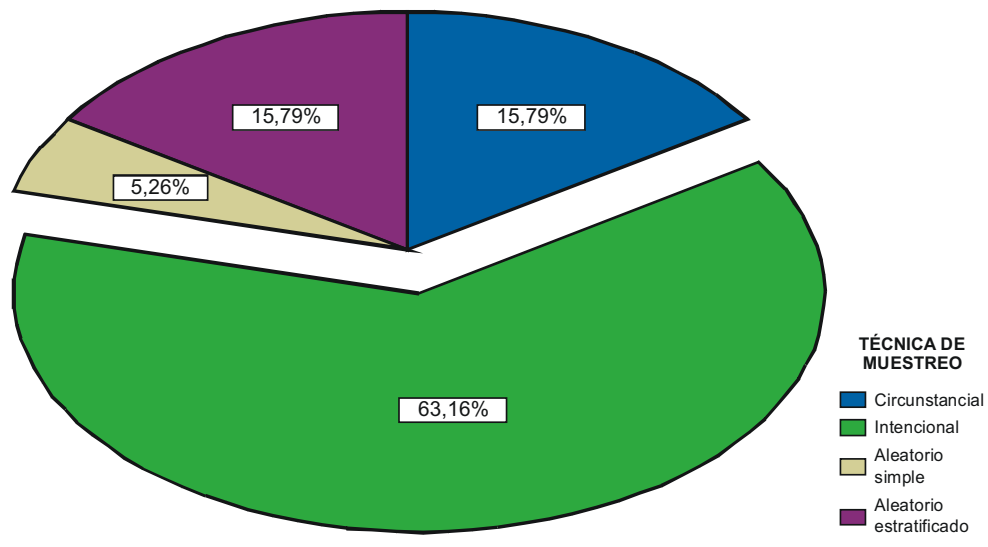

\section{Procesamiento y análisis de la información}

En referencia a las Técnicas estadísticas utilizadas en la investigación, datos estimados con una significación estadística de $\times^{2}=0.000$, podemos observar (Gráfico 10) una evidente predominancia de las técnicas estadísticas inferenciales $74 \%$ sobre las demás técnicas estadísticas. 
Gráfico 10. Análisis estadístico de la investigación.

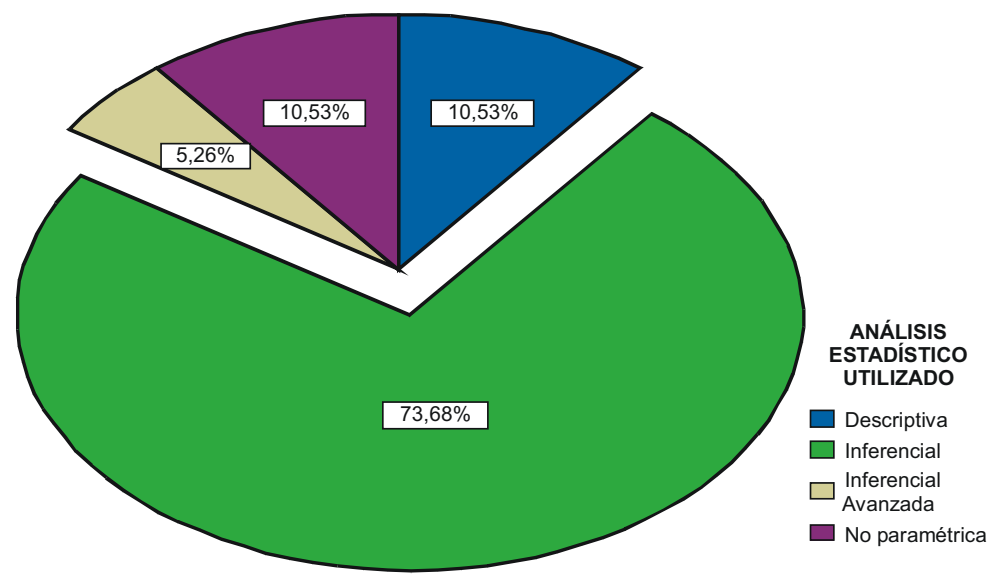

\section{Referencias que fundamentan las tesis de investigación}

A este respecto es conveniente iniciar este apartado con el mapa conceptual de la Figura 3 que ilustra las relaciones conceptuales que el instrumento establece entre los tipos de referencia y los idiomas en que están escritas.

Figura 3. Tipos de idiomas de las referencias a las fuentes de información.

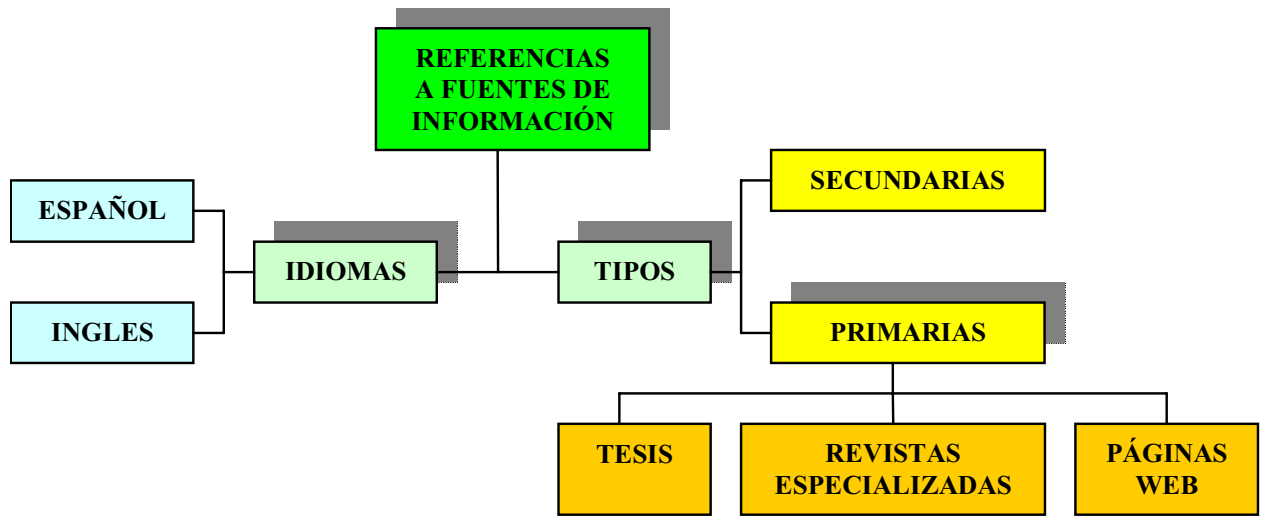

En relación a las fuentes de información primarias y secundarias utilizadas en las tesis investigación, datos estimados con una significación estadística de $\times^{2}=0.010$, podemos observar (Gráfico 11) una llamativa predominancia de las referencias secundarias $69.41 \%$ sobre las primarias $30.59 \%$. 
Gráfico 11. Pocentaje de las referencias primarias y secundarias en las tesis.

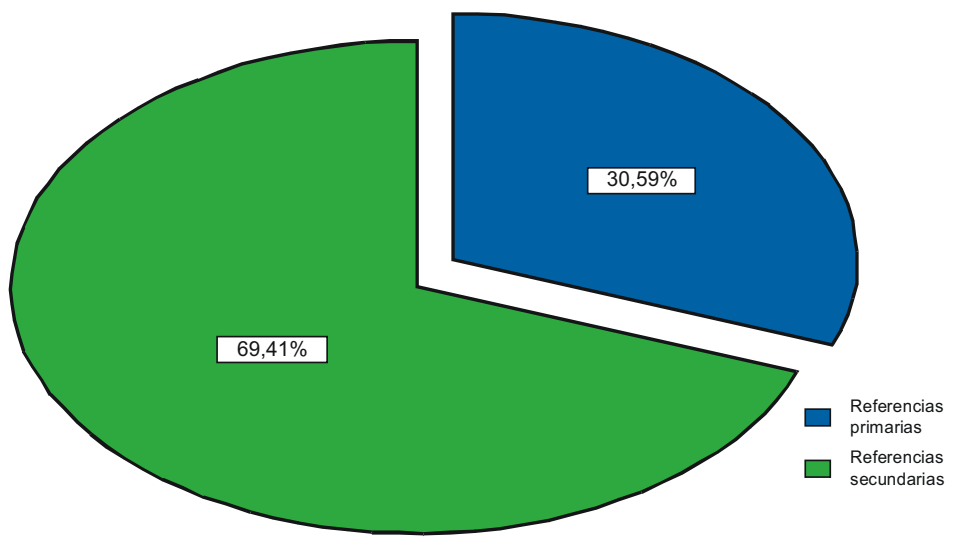

En relación a las fuentes de información en español y en inglés utilizadas en las tesis de investigación, datos estimados con una significación estadística de $\times^{2}=0.000$, podemos observar (Gráfico 12) una evidente predominancia de las referencias en español $77.13 \%$ sobre las referencias en inglés $22.87 \%$.

Gráfico 12. Pocentaje de las referencias en idiomas español e inglés en las tesis.

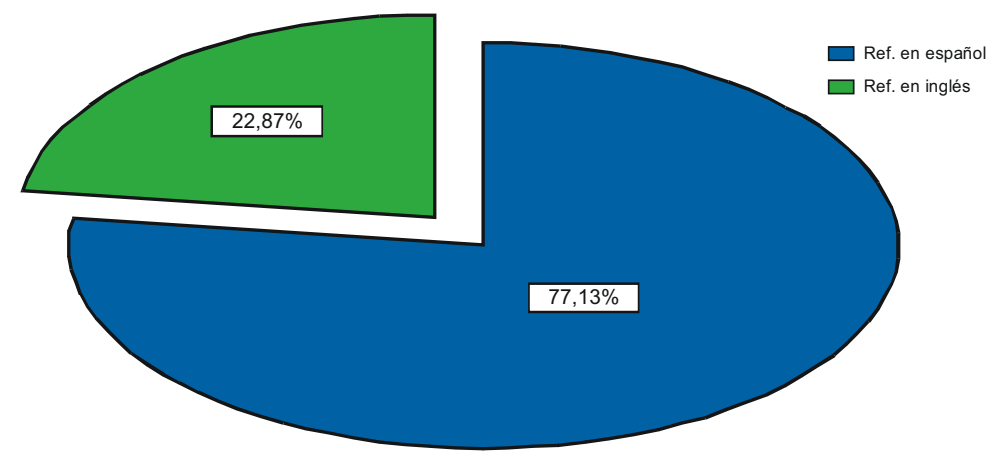

\section{Poblaciones estudiadas}

Es conveniente comenzar este apartado con el mapa conceptual de la Figura 4 que ilustra las relaciones conceptuales que el instrumento establece entre los distintos indicadores poblacionales. 
Figura 4. Características de las poblaciones estudiadas consideradas en este estudio.

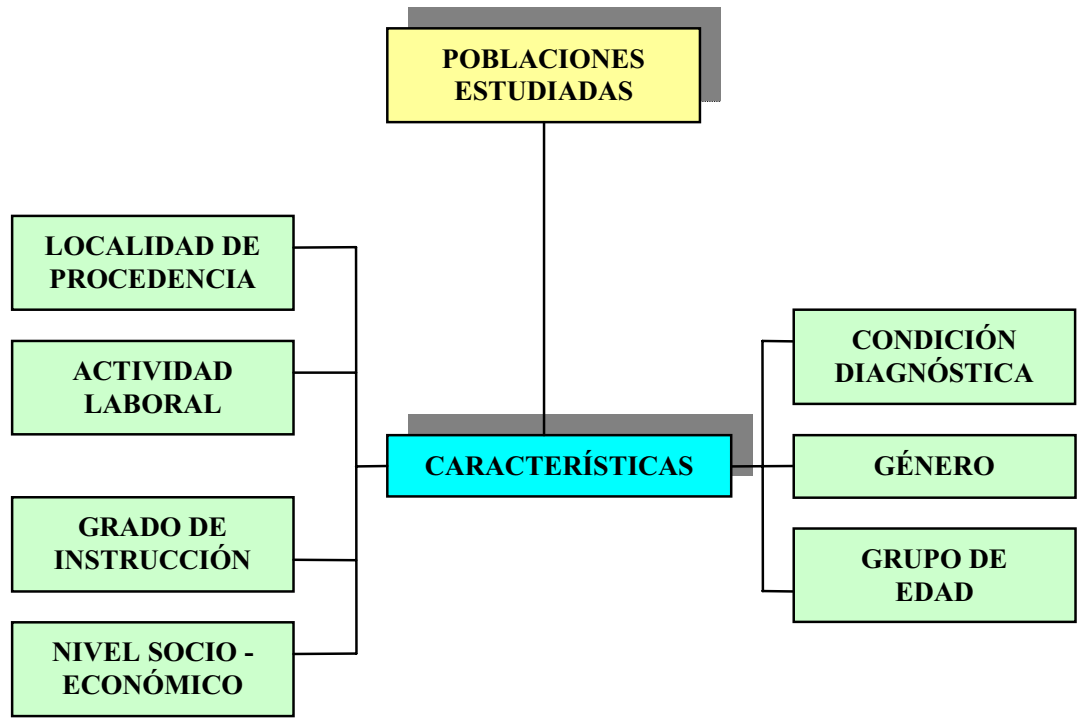

En cuanto a los gráficos correspondientes a cada una de las características poblacionales nos dispensamos de comentarlos por ser su interpretación autoevidente.

Gráfico 13. Sujetos investigados según su condición diagnóstica.

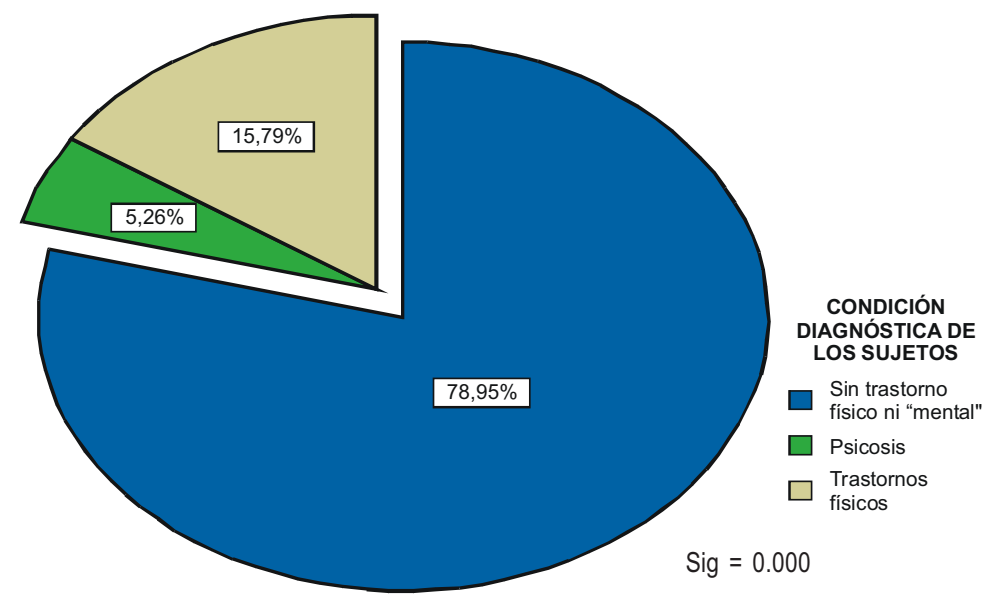


Gráfico 14. Sujetos investigados según su condición laboral.

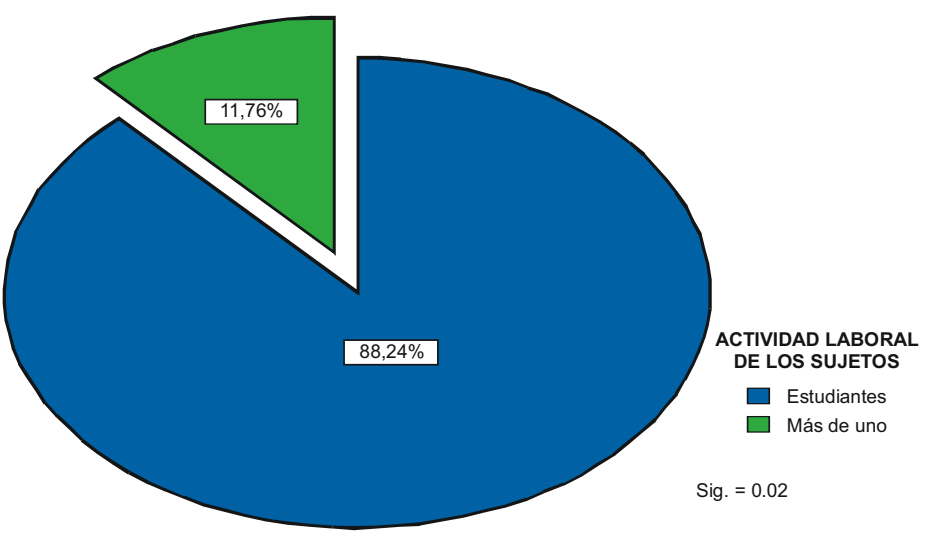

Gráfico 15. Sujetos investigados según su grado de instrucción.

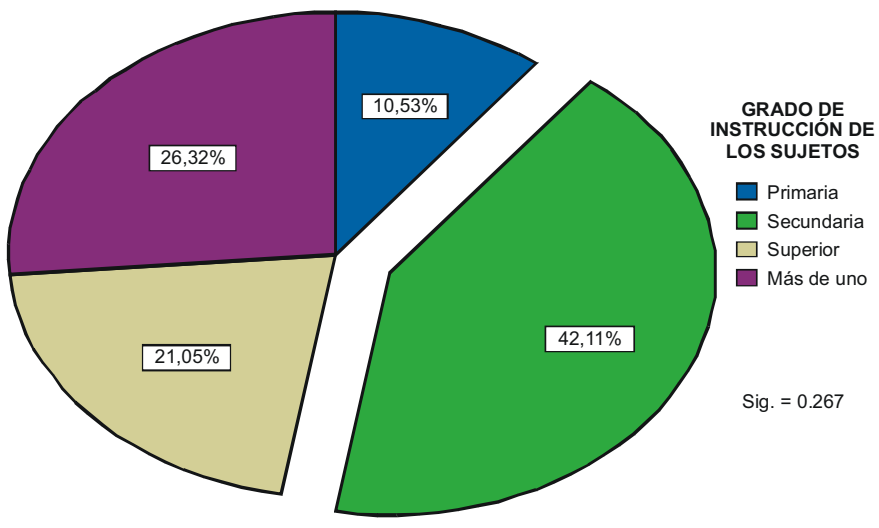

Gráfico 16. Sujetos investigados según su edad.

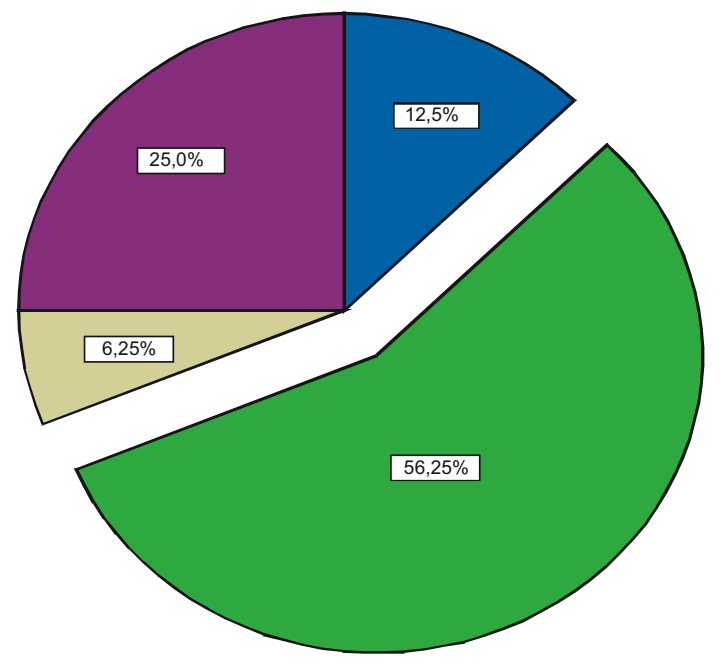

GRADO DE EDAD

DE LOS SUJETOS

$\square$ Niños

$\square$ Adolescentes

$\square$ Adultos

$\square$ Más de uno

Sig. $=0.023$ 
Gráfico 17. Sujetos investigados según su localidad de procedencia.

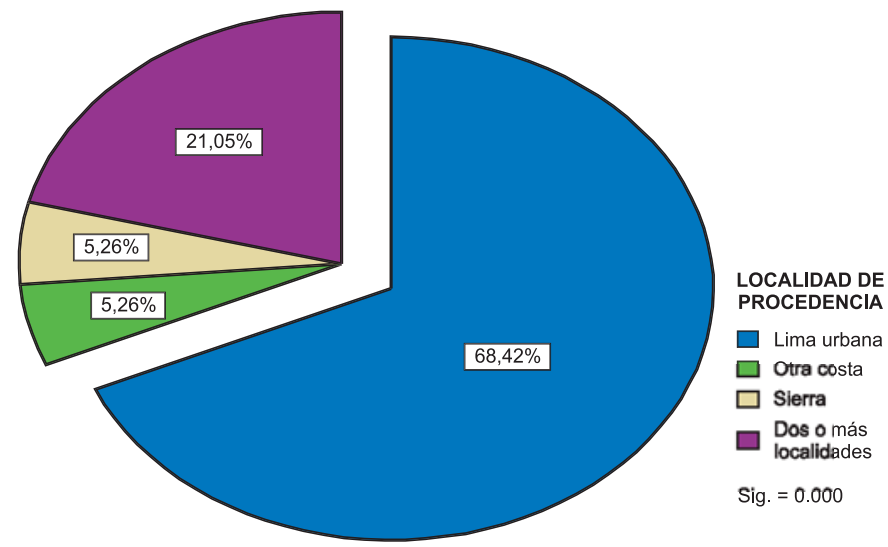

Gráfico 18. Sujetos investigados según su género.

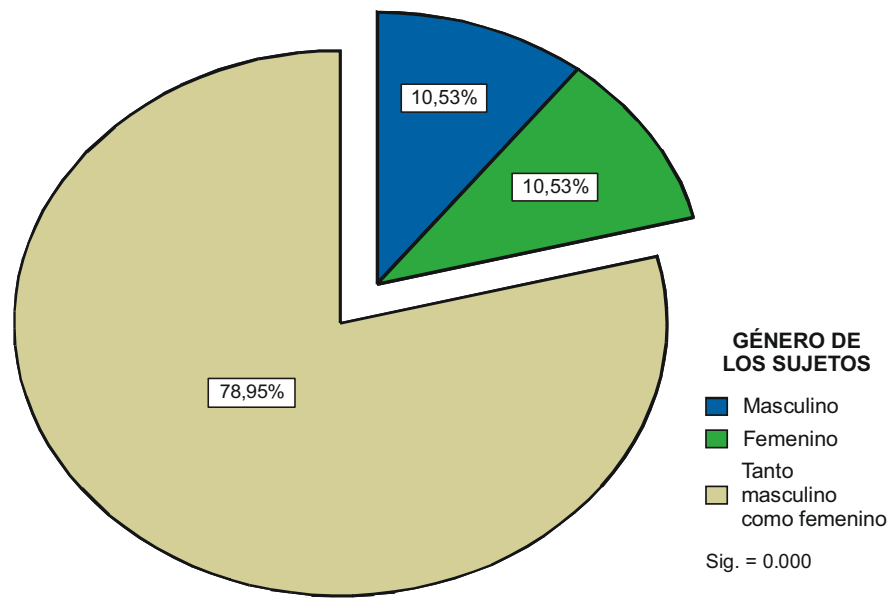

Gráfico 19. Sujetos investigados según su condición socioeconómica.

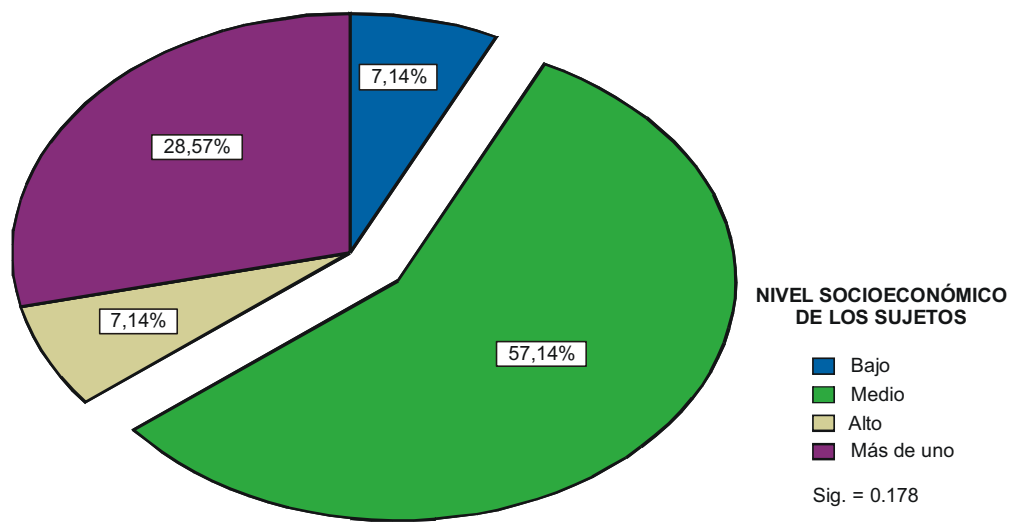




\section{CONCLUSIONES}

1. En el corpus de datos predominan las tesis de Investigación, estando constituidas en mayor parte por investigaciones Básicas Fácticas.

2. Los procesos psicológicos básicos, desarrollando procedimientos de intervención, han sido estudiados sólo desde el enfoque cognitivo-conductual.

3. La violencia Familiar ha sido estudiada sólo desde el enfoque psicosocial y con una metodología descriptiva.

4. Como técnica de selección de las muestras poblacionales a investigar predomina el muestreo intencional.

5. Destaca el análisis de los datos con estadística inferencial.

6. Las poblaciones estudiadas se caracterizan por ser estudiantes adolescentes tanto mujeres como varones procedentes de Lima urbana que no adolecen de trastorno físico ni mental.

7. Por último, en cuanto a las fuentes informativas que fundamentan las investigaciones se observa una predominancia de las referencias secundarias y en español, y una correlación significativa entre el total de referencias y las referencias tanto primarias, como secundarias, así mismo se constatan correlaciones significativas entre las referencias en español y las referencias primarias, secundarias y el total de referencias

\section{REFERENCIAS BIBLIOGRÁFICAS}

1. Alcaide, R. (1999). «Las publicaciones sobre higienismo en España durante el período 1736-1939: un estudio bibliométrico». Scripta Nova. Revista Electrónica de Geografía y Ciencias Sociales. Universidad de Barcelona, Vol. III No 37, 1 de abril de 1999.(http:/ /www.ub.es/geocrit/sn-37.htm)

2. Algaba, A. (2000). «La difusión de la innovación. Las revistas científicas en España 1760-1936». Scripta Nova. Revista Electrónica de Geografía y Ciencias Sociales. Universidad de Barcelona, Vol. IV, Nº 69 (27), 1 de agosto de 2000. (http://www.ub.es/ geocrit/sn-69-27.htm)

3. Arnau, J. (1986). Diseños Experimentales en Psicología y Educación. México: Trillas

4. Carpintero (1981). «La 'ciencia de la ciencia' y la investigación psicológica en el mundo contemporáneo». En Carpintero y Peiro (Eds), Psicología Contemporánea. Teoría y Metodos cuantitativos para el estudio de su literatura científica. Valencia: Alfaplas.

5. Carpintero, H. y Peiro, J. (1981). «Una perspectiva bibliométrica sobre la Modificación de Conducta». En Carpintero y Peiro (Eds), Psicología Contemporánea. Teoría y Métodos cuantitativos para el estudio de su literatura científica. Valencia: Alfaplas

6. Carpintero, H. y Tortosa, F. (1990). Aplicaciones de la metodología bibliométrica a la historia de Psicología: una revisión de conjunto. En: F. Tortosa, L. Mayor y 
H. Carpintero (dirs.). La Psicología contemporánea desde la Historiografía. Barcelona: PPU.

7. Castro, L. (1980). Diseño Experimental sin Estadística. México:Trillas

8. Hernández, R., Fernández, C. y Baptista, P. (1996). Metodología de la investigación. Bogotá, Mc Graw Hill.

9. Kerlinger, F. Y Howar, L. (2001). Investigación del Comportamiento. México: Pretince Hall.

10. Escuela Académico Profesional de Psicología (1996). Currículum de Pregrado para la Profesión de Psicólogo. Lima: Facultad de Psicología de la UNMSM

11. Escuela Académico Profesional de Psicología (1993). Reglamento de Grados y Títulos para la Facultad de Psicología. Lima: Facultad de Psicología de la UNMSM.

12. Gardfield, E. (1995). Análisis Cuantitativo de la literatura científica y sus repercusiones en la formulación de políticas científicas en América Latina y el Caribe. Boletín Oficina Sanit. Panam. 118 (5) pp.448-455

13. Garfield, E. et al. (1978). Citation data as science indicators. En: Y. Elkana et al., Towards a metric of science. New York: Wiley

14. Maura, M., Mercado, J. y Aristeguieta, S. (2004). «Las tesis de maestría de la Escuela Graduada de Ciencias y Tecnologías de la Información (EGCTI): un estudio bibliométrico». En: Simbiosis, Vol.1 No1, (http://egcti.upr.edu/simbiosis/ Vol\%201\%20Num\%201/ArtMaura.htm)

15. Price, D. (1973) Hacia una ciencia de la ciencia. Barcelona. Ariel.

16. Price, D. (1978) «Towards a model for science indicators». En: I. Elkana et al., Towards a metric of science. New York: Wiley.

17. Pulgarín, A., Carapeto, C. y Cobos, J. (2004). Análisis bibliométrico de la literatura científica publicada en "Ciencia. Revista hispano-americana de ciencias puras y aplicadas» (1940-1974). En: Information Research. Vol. 9, No 4. (http://informationr.net/ ir/9-4/paper193.html)

18. Quintana, A. (1995). Propuesta de un formato de Observación de Reportes de Investigación. Boletín de Post-Grado Psicología. UNMSM. Año V. No 4. pp. 03-13.

19. Quintana, A. (1996). «Un Modelo de Aproximación Empírica a la Investigación en Psicología y Ciencias Humanas: El Caso de la Investigación en Anales de Salud Mental». Revista Peruana de Psicología. C. Ps. P.- C.D.N. Año 1, Vol. 1. Nº 1. pp. $07-25$

20. Quintana, A. y Ojeda, G. (1996). «El análisis neobibliométrico: un aporte al desarrollo de una epistemología empírica». En: Facetas. C. Ps. P.-C.D.R.-Lima, Vol. 1, No 02. pp. 35-40. 
